\title{
Variation in flowering phenology and selfing rate across a contact zone between diploid and tetraploid Arrhenatherum elatius (Poaceae)
}

\author{
CHRISTOPHE PETIT* ${ }^{*}$ PHILIPPE LESBROS, XUEJUN GE $†$ \& JOHN D. THOMPSON \\ Centre d'Ecologie Fonctionnelle et Evolutive, C.N.R.S., Route de Mende, BP 5051, F-34033 Montpellier, France
}

\begin{abstract}
Plant populations with different ploidy levels have frequently been found to develop mechanisms of reproductive isolation when they occur in contact, presumably because of selection against the production of inviable or sterile hybrids. A parapatric contact zone between diploid and tetraploid Arrhenatherum elatius has been identified in the Spanish Pyrenees. Of 145 flowering plants sampled in the zone one was triploid. This study provides data on enzymatic allele frequencies, flowering time and selfing rate across the contact zone, to examine whether reproductive isolation occurs to limit the potential production of triploids. To substantiate these data, flowering time was measured in plants grown in a common garden and outcrossing rates were compared to those in one allopatric population of each cytotype from an environmentally similar habitat. Nei's genetic distances calculated on the basis of allele frequencies showed no clear evidence for past hybridization and/or introgression between diploids and tetraploids. Patterns of flowering phenology in the field and in controlled conditions indicated almost total flowering divergence between parapatric cytotypes; this divergence may be under genetic control. There was, however, no evidence of current phenotypic selection on flowering date in the contact zone. For each cytotype, multilocus selfing rates were higher in parapatric populations than in allopatric populations. The possibility that flowering time divergence and increased selfing have evolved in the contact zone resulting in reproductive isolation is discussed.
\end{abstract}

Keywords: flowering phenology, hybridization, polyploidy, reproductive isolation, selfing rate.

\section{Introduction}

The role of reproductive isolation in the coexistence of closely related sympatric and parapatric taxa is a fundamental issue in evolutionary biology. Reproductive isolation can be either prezygotic or postzygotic. Prezygotic barriers to mating between taxa include ecological (adaptation to different microhabitats in which most matings occur), phenological (mating occurs at different times), behavioural (no mating occurs among taxa), or physiological (male gamete transfer is blocked by some form of crossincompatibility). Postzygotic barriers include hybrid inviability and sterility. Because natural selection

\footnotetext{
${ }^{*}$ Correspondence. E-mail: petit@cefe.cnrs-mop.fr

†Present address: South China Institute of Botany, 510650 Academia Sinia, Guangzhou, China.
}

only acts on prezygotic factors to isolate taxa in contact, there has been much interest in how prezygotic reproductive isolation may isolate taxa and 'reinforce' any pre-existing postzygotic barriers (Dobzhansky, 1940; Howard, 1993).

In plants, reproductive contact between closely related taxa represents an ideal situation for examining how divergence of reproductive traits may evolve. Gametic wastage and reduced hybrid fitness have been suggested to explain divergence in reproductive traits such as flowering time (Van Dijk \& Biljsma, 1994) and increased selfing (Levin, 1985). In recent years there has been much interest in the coexistence of diploid and related tetraploid plants which, because of triploid block (endosperm inviability) and hybrid sterility or inviability, must show some form of habitat or reproductive isolation in order to coexist (Levin, 1975; Felber, 1991; Thomp- 
son \& Lumaret, 1992). For example, in the polyploid complex Dactylis glomerata, field studies (Lumaret \& Barrientos, 1990) and controlled experiments (Bretagnolle \& Thompson, 1996) have illustrated how a combination of microhabitat and phenological differences may facilitate reproductive isolation and coexistence of diploid and tetraploid plants in a natural sympatric situation. In Plantago media, simulation models suggest that diploid and tetraploid coexistence is facilitated by the development of a mosaic of parapatric zones of single cytotypes (Van Dijk \& Biljsma, 1994). In P. media the degree of separation in flowering time between plants of different cytotypes is also positively correlated with seed set within each cytotype, indicating that selection may act on such variation in flowering time (Van Dijk, 1991). Elsewhere it has been shown that natural selection can shape patterns of flowering phenology (Zimmerman \& Gross, 1984), hence the possibility that such variation reflects contemporary patterns of selection.

Increased self-compatibility may also be a factor facilitating coexistence among closely related taxa and reducing gene flow among locally adapted populations. Increased self-compatibility has been suggested to facilitate reproductive isolation in zones of sympatry between different species of Phlox (Levin, 1985) and Calyptridium (Hinton, 1976), and as a barrier to gene flow from nontolerant populations into heavy-metal tolerant populations of the grass Anthoxanthum odoratum (Antonovics, 1968). Similarly in Armeria maritima, Lefèbvre (1970) reported high levels of self-fertility in heavy-metal tolerant plants. In Arrhenatherum elatius, Cuguen et al. (1989) suggested that low levels of inbreeding depression may favour the evolution of selfing in plants on contaminated soils. However, no attention has yet been paid to the potential role of increased selfing in diploid-tetraploid contact zones as a mechanism favouring reproductive isolation.

In this study we report an initial investigation of variation in flowering time and selfing rates across a diploid-tetraploid contact zone in the perennial grass Arrhenatherum elatius. The contact zone comprises an altitudinal gradient with diploids predominantly in natural habitats at high altitude and tetraploids in lower altitude human-disturbed habitats. Previous observations have shown that a small, but not negligible, proportion (about 1 per cent) of triploids is produced in the zone (Petit \& Lesbros, unpublished data). The objectives of this study are as follows. First, we document the spatial location of the different ploidy levels across the contact zone from 1200 to $1800 \mathrm{~m}$. Secondly, we examine flowering time in plants across the zone in situ and subsequently grown in an experimental garden. Thirdly, we estimate selection differentials and selection gradients using the multiple regression approach of Lande \& Arnold (1983) to examine whether natural selection may be acting on flowering time variation in diploids and tetraploids across the contact zone. Finally, we quantify selfing rates in parapatric and allopatric diploid and tetraploid populations.

\section{Materials and methods}

\section{Study species and site}

Arrhenatherum elatius (L.) J. \& C. Presl. (Poaceae) is a perennial grass composed of diploid and tetraploid subspecies. The species is mainly outcrossed and wind-pollinated, but previous observations have shown that at least the subspecies elatius is partly self-compatible (Sulinowski, 1965; Cuguen et al., 1989; Thompson \& Ge, unpublished data).

In this study we focus on the diploid subspecies sardoum and the tetraploid subspecies elatius. The diploid subspecies sardoum occurs in pine forests and on dry scree slopes of montane areas of Spain, Corsica and Sardinia. The tetraploid elatius is widely distributed throughout Europe over a wide range of ecological conditions from low altitude plains up to altitudes of $3000 \mathrm{~m}$ in the Caucasus mountains (see Pfitzenmeyer, 1962). In the central Spanish Pyrenees, the diploid sardoum and the tetraploid elatius occur in parapatry along an altitudinal gradient. The diploids occur on medium-high altitude $(>1000 \mathrm{~m})$ scree slopes whereas the tetraploid is common in highly human-disturbed sites up to $1500 \mathrm{~m}$. Tetraploid populations are often very close $(<100 \mathrm{~m})$ to diploid populations. At one site on El Turbon mountain (Aragon, Spain), diploids and tetraploids have been found less than $20 \mathrm{~m}$ apart. This site is the focus of the present study.

\section{Sampling and determination of different ploidy levels}

To map the spatial location of different ploidy levels at the El Turbon site we labelled a total of 248 plants with number-coded white sticky tape in two main ecological zones. One hundred and twenty-four plants were sampled at $1200-1400 \mathrm{~m}$ on field margins and along the road through the village of Vilas del Turbon (roadside zone), and 124 plants were sampled on two scree slopes between 1500 and $1800 \mathrm{~m}$ (scree slope zone). At the end of the 
growing season, all the surviving plants were transplanted to the CEFE-CNRS experimental garden in Montpellier (France). When new leaves appeared on each plant in the experimental garden, the ploidy level of each genotype was determined by flow cytometry. Diploid and tetraploid plants on which chromosome counts had previously been made were used as controls. Several labelled plants died or were not found at the end of the study, particularly in the roadside zone. A total of 145 plants, 86 from the scree slope zone and 59 from the roadside zone, flowered and survived in 1994. Flow cytometry revealed 39 tetraploid, 19 diploid (hereafter RS4 and RS2, respectively) and one triploid plant in the roadside zone (Fig. 1) whereas the 86 plants from the scree slope zone (hereafter SZ2) were all diploid. A second triploid plant was found in the roadside zone but did not flower in the year of the experiment.

To quantify selfing rates and to assess the genetic component of flowering phenology in controlled conditions, two allopatric populations, one diploid and one tetraploid, both in situations completely isolated from the other ploidy level, were also chosen for study. These two populations occur in similar habitats to the parapatric populations in the same region. The tetraploid population occurs at $800 \mathrm{~m}$ on the edge of a pine forest near Santa Cruz de la Seros (SCS) at $100 \mathrm{~km}$ estimated map distance from El Turbon, and the diploid population on a scree slope at $1500 \mathrm{~m}$ in the Sierra de Guara (SGA), at $60 \mathrm{~km}$ estimated map distance from El Turbon. Ploidy levels of plants in allopatric populations were confirmed using flow cytometry (see above).

\section{Flowering phenology in natural populations and in controlled conditions}

Date of initial flowering (DIF) and flowering duration (FD) were quantified at the El Turbon site on each of the 248 marked plants. To quantify flowering phenology eight visits were made to the site on the $15 / 05,14 / 06,27 / 06,1 / 07,7 / 07,12 / 07,19 / 07$ and 22/07 in 1994.

In order to test whether flowering phenology may have a genetic component, 10 diploids from the scree slope zone, 10 diploids and 10 tetraploid genotypes from the roadside zone, and 10 genotypes from each of the two allopatric populations (SGA and SCS) were transplanted to the CEFE-CNRS experimental garden at Montpellier (France), in autumn 1994. Three replicate cuttings of standard size comprising a single rooted tiller were then grown in two environmental treatments: either full sunlight or 50 per cent sunlight. This gave a total sample size of five populations $\times 10$ genotypes $\times 2$ environments $\times 3$ blocks $=300$ plants. This was part of a larger experiment designed to analyse relative levels of phenotypic plasticity of diploid and tetraploid populations of this species sampled in open and shaded habitats. The SGA plants were sampled on open scree slopes, and SCS along trail sides in a pine forest. DIF and FD for each plant were quantified by observation every three or four days from April to July 1995.

ANOVAS were performed in each of the two experimental environments to detect differences among populations in DIF and FD. Since only three genotypes of SGA flowered in environment 2 (50 per cent sunlight), this population-environment combination was not included in the analyses.

\section{Phenotypic selection on flowering phenology}

To examine whether variation in flowering time is correlated with reproductive success we calculated selection differentials and gradients for DIF and FD of diploid and tetraploid plants across the contact zone, using a multiple regression approach (Lande \& Arnold, 1983). Phenological data were collected as described above, and genotypic reproductive success in 1994 was estimated as the mean number of seeds per ramet on the same plants. Trait values were standardized to mean $=0$ and variance $=1$ in order to allow direct comparisons among character and genotype samples.

Selection differentials were calculated by means of Pearson's correlation coefficients between the mean phenotypic trait of each genotype and its relative reproductive success (Lande \& Arnold, 1983), using PROC CORR in SAS (1990). Relative reproductive success was estimated as the mean number of seeds per ramet produced by each genotype weighted by the mean number of seeds per ramet calculated in the group of individuals included in each analysis. Selection differentials calculated in this way determine indirect selection (selection on correlated characters) and the direct effects of selection on the traits studied. Selection gradients, which only provide evidence for a direct effect of selection, were calculated by means of multiple linear regression (Lande \& Arnold, 1983) with PROC REG (linear selection) and RSREG (nonlinear selection) in SAS (1990). Linear (directional) selection is determined by the covariation between the mean trait value and relative fitness whereas nonlinear (including stabilizing-disruptive) selection is calculated by means of quadratic analyses using squared data of 
the mean trait value and the relative genotypic fitness (Lande \& Arnold, 1983). Because multiple correlation analysis increases the probability of a type I error, the significance level of all correlations in each group of individual analyses was corrected using the sequential Bonferroni correction. As high correlations (multicollinearity) between measured characters may not be compatible with selection gradient estimation (Mitchell-Olds \& Shaw, 1987), multicollinearity was estimated with the variance inflation ratio (VIF) option in PROC REG in SAS (1990). Multicollinearity was not detected, and hence the two phenological traits were retained. Selection differentials and gradients were calculated in this way for diploids in the scree slope zone, and for both tetraploids and diploids in the roadside zone. Because the sample size of diploids in the roadside zone (RS2) was small $(n=19)$, selection gradients could not be estimated, hence only selection differentials were calculated for this group of plants.

Because true fitness functions may be more complex than the smooth functions estimated by parametric regressions, results of linear regression were corroborated by nonparametric estimations of the fitness functions (using the cubic spline approach) with the GLMS computer software of Schluter (1990). This method does not depend on an a priori model of phenotypic distributions.

\section{Estimation of selfing rates}

Selfing rates in allopatric (SCS and SGA) and parapatric (RS4 and SZ2) diploid and tetraploid popula- tions were calculated from electrophoretic analyses of leaf material from nine seedlings from each of 15 naturally pollinated maternal plants in each population. Seeds were collected in summer 1993 and germinated in winter 1994. Leaf material was collected from the seedlings in spring 1994. Enzymatic electrophoreses were performed using starch gels and carried out on three polymorphic loci: glutamic-oxaloacetic transaminase (GOT, EC 2.6.1.1), phosphoglucoisomerase (PGI, EC 5.3.1.9) and leucine aminopeptidase ( $L A P$, EC 3.4.11.1). Several other loci were assayed but showed no readily interpretable banding patterns, particularly for the polyploid plants. The ploidy level of all seedlings was verified by flow cytometry. Single locus $\left(t_{\mathrm{s}}\right)$ and multilocus $\left(t_{\mathrm{m}}\right)$ outcrossing rates were calculated using the computer software MLT (for diploids) and MLTET (for tetraploids) from Ritland (1990, 1991). Standard errors for the population level estimates of outcrossing rate were determined from 500 bootstraps for each population. Biparental inbreeding can then be calculated as $\left(t_{\mathrm{m}}-t_{\mathrm{s}}\right)$.

\section{Genetic distance among populations}

Nei's (1978) unbiased genetic distances between the two parapatric (RS4 and SZ2) and the two allopatric (SCS and SGA) populations were calculated from allelic frequencies at the three enzymatic loci described above, using the Blosys-1 program (Swofford \& Selander, 1981). Twenty seedlings (each from a different mother plant) in each of the four populations were randomly chosen to estimate allelic frequencies in the four populations.

Table 1 Mean values $( \pm$ SE) for phenological, morphological and reproductive traits for diploids and tetraploids in the natural parapatric populations of Arrhenatherum elatius on El Turbon mountain

\begin{tabular}{lcccc}
\hline & \multicolumn{2}{c}{ Diploids } & Tetraploids \\
\cline { 2 - 3 } & $\begin{array}{c}\text { Scree slope zone (SZ2) } \\
(1500-1800 \mathrm{~m}) n=86\end{array}$ & $\begin{array}{c}\text { Roadside zone (RS2) } \\
(1200-1400 \mathrm{~m}) n=19\end{array}$ & $\begin{array}{c}\text { Roadside zone (RS4) } \\
(1200-1400 \mathrm{~m}) n=39\end{array}$ \\
\hline Date of initial flowering & & & \\
$\quad$ (days after 15/5/94) & $43.63(0.19)$ & $45.64(1.02)$ & $31.80(0.86)$ \\
Flowering duration (days) & $7.87(0.43)$ & $9.64(0.68)$ & $12.55(0.44)$ \\
Stem height (cm) & $47.18(1.03)$ & $53.29(2.38)$ & $89.64(3.05)$ \\
Panicle length (cm) & $12.57(0.25)$ & $12.29(0.47)$ & $21.32(0.70)$ \\
Leaf width (mm) & $1.86(0.07)$ & $1.71(0.14)$ & $5.20(0.23)$ \\
Leaf length (cm) & $5.13(0.18)$ & $5.01(0.49)$ & $10.86(0.49)$ \\
Number of seeds per ramet & $11.35(0.71)$ & $7.52(2.05)$ & $27.85(3.59)$ \\
Total seed weight (mg) & $13.33(0.91)$ & $11.76(2.45)$ & $82.00(12.96)$ \\
\hline
\end{tabular}

Altitudes of sampled plants are in parentheses. 


\section{Results}

\section{Flowering phenology in natural conditions}

Mean values for each trait are reported in Table 1. Diploids and tetraploids showed significant differences for their average time of initial flowering ( $t$-test: $t=13.55, P<0.0001$ and $t=9.93, P<0.0001$ for SZ2-RS4 and RS2-RS4 comparisons, respectively). The flowering time of diploid plants did not vary significantly between the roadside and scree slope zones $(t=-1.73, P=0.1008$ for the RS2-SZ2 comparison). All but two tetraploid genotypes

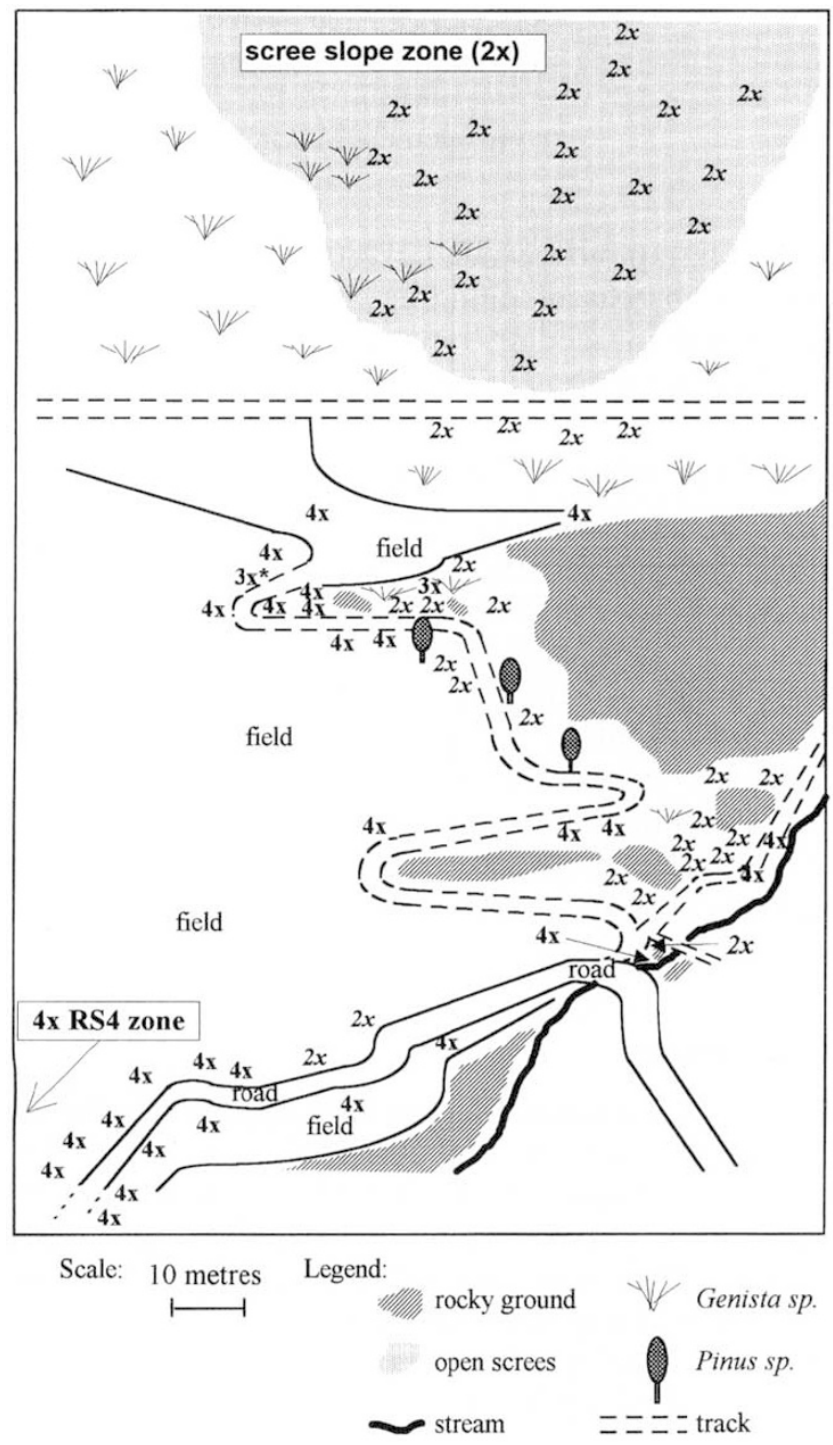

Fig. 1 Schematic map of the distribution of diploid, triploid and tetraploid Arrhenatherum elatius across the contact zone at the El Turbon study site (altitude $1380-1500 \mathrm{~m}$ ). $4 \mathrm{x}$ zone: part of the roadside zone (RS) where only tetraploids were found. $3 \mathrm{x}^{*}$ : nonflowering triploid plant. completed flowering before any of the diploid plants began to flower (Fig. 2). Flowering duration was also significantly longer for tetraploids compared to diploids from the scree slope zone $(t=-7.80$, $P<0.0001)$ and from the roadside zone $(t=-3.72$, $P=0.0006)$. Diploids from the two zones showed significant differences in flowering duration with plants from the roadside zone having a significantly longer flowering period $(t=-2.09, P=0.0393)$. The triploid genotype flowered later than the diploids (Fig. 2) but did not set seed.

\section{Flowering phenology in controlled conditions}

Significant differences in the date of initial flowering between parapatric diploid (SZ2: mean date of initial flowering $=96.75( \pm 1.25)$ days and 95.75 $( \pm 2.21)$ days in full sunlight and 50 per cent sunlight, respectively) and tetraploid (RS4: 77.63 $( \pm 2.92)$ days and $73.76( \pm 1.93)$ days) plants were observed in controlled conditions (Fig. 3) $(F=104.08, P<0.0001$, and $F=4165.17, P<0.0001$ for full sunlight and 50 per cent sunlight environments, respectively). Allopatric tetraploids (SCS: $87.60( \pm 2.46)$ days) and diploids (SGA: 107.55 $( \pm 4.24)$ days $)$ showed a slight but significant ( $F=299.56, P=0.0002$ ) overlap in the full sunlight environment (Fig. 3). In contrast to field data, flowering duration was not significantly different between parapatric diploids $(7.90( \pm 0.57)$ days and $7.53( \pm 0.69)$ days in full sunlight and 50 per cent sunlight environments, respectively) and tetraploids (11.66 $( \pm 2.48)$ days and $9.13( \pm 1.02)$ days $)$ in the two environments studied $(F=4.22, P=0.1762$ and $F=1.41, P=0.3574$ for the full sunlight and 50 per cent sunlight environments, respectively).

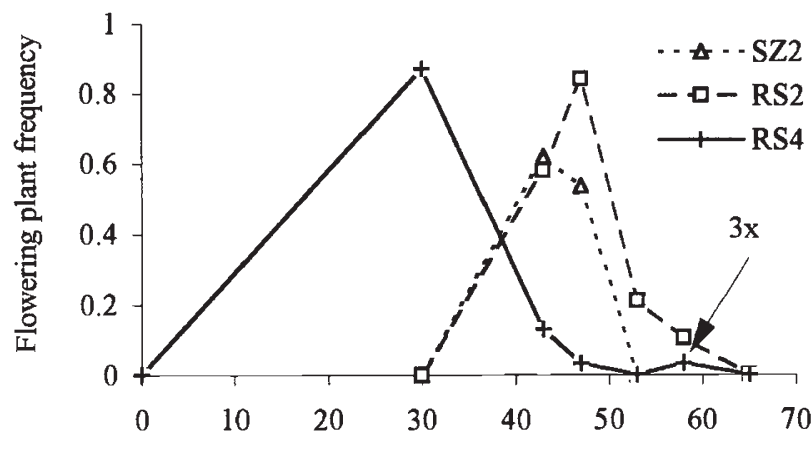

Date (in number of days from 15 May 1994)

Fig. 2 Phenology of flowering in diploids, triploid and tetraploids of Arrhenatherum elatius in the contact zone at El Turbon. 


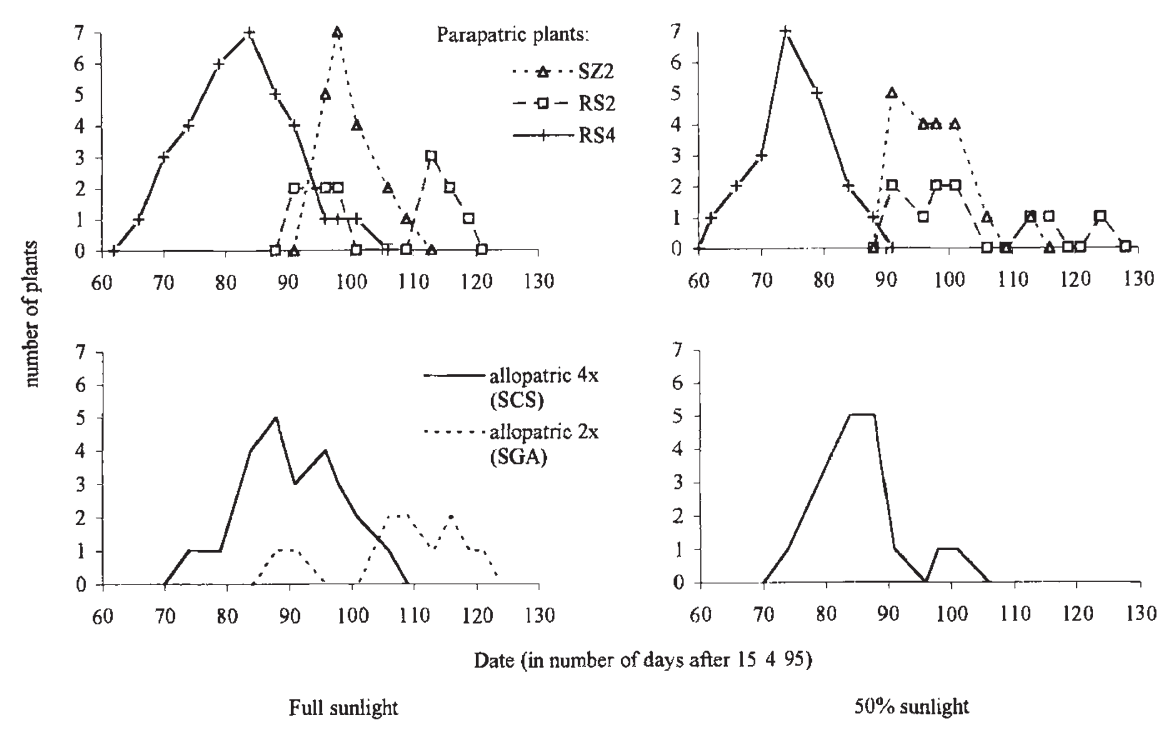

Fig. 3 Phenology of flowering time in parapatric and allopatric (SGA and SCS) diploids and tetraploids of Arrhenathenum elatius grown in two controlled environments.

\section{Phenotypic selection on flowering phenology}

Scree slope zone diploids showed a significantly greater mean seed number than roadside zone diploids ( $t$-tests: $t=2.14 ; P=0.0345$ ) but an equal total seed weight $(t=0.85 ; P=0.3924)$. Tetraploids produced significantly more seeds per ramet than diploids from the scree slope zone $(t=-4.50$; $P=0.0007)$ and the roadside zone $(t=-4.91$; $P=0.0006$ ). Total seed weight was significantly greater for tetraploids than diploids $(t=-5.28$; $P<0.0001$ and $t=-5.36, P<0.0001$ for RS4-SZ2 and RS4-RS2 comparisons, respectively).

Phenotypic selection analyses showed no evidence of correlated variation between phenological characters and relative estimated fitness in parapatric tetraploids and in diploids. These results were confirmed by nonparametric estimations of fitness functions (the results are not shown but are available on request from the senior author).

\section{Selfing rates}

Isozyme electrophoresis revealed four and three alleles for GOT, three and two for PGI, and five and three alleles for LAP in tetraploids and diploids, respectively (Table 2). No diagnostic alleles were found to discriminate among the cytotypes in the contact zone. The multilocus and single-locus outcrossing rates for each cytotype were found to be lower in the parapatric populations compared to the allopatric populations (Table 3). Standard errors (SE) associated with these outcrossing rates indicate that the difference between parapatric and allopatric populations was significant for the diploids but not for the tetraploids, which nevertheless show a similar trend of increased selfing in parapatric populations. Biparental inbreeding rates $\left(t_{m}-t_{s}\right)$ were extremely low in all four populations (Table 3 ).

\section{Genetic distance among populations}

Nei's genetic distance (1978) estimates based on allele frequencies for the three polymorphic loci described above show greater genetic similarity between parapatric and allopatric tetraploid populations (mean genetic distance $=0.042$ ) than between diploid populations (mean genetic distance $=0.067$ ). Nevertheless, allelic frequencies and diversities show no evidence for introgression or hybridization between parapatric tetraploids and diploids (mean genetic distance $=0.083$ ) in the contact zone (Table 2).

\section{Discussion}

Prezygotic isolation mechanisms appear to play an important role in the restriction of gene flow between diploids and tetraploids of Arrhenatherum elatius in the contact zone at El Turbon. Our study indicates that both flowering time divergence and increased selfing may act to limit potential hybridization between the parapatric (and sympatric) diploids. The significance of each of these mechanisms is now discussed.

\section{Flowering time divergence across the diploid- tetraploid contact zone}

Populations in the parapatric zone showed marked differences in flowering phenology both in the field 
Table 2 Allelic frequencies at three enzymatic loci in parapatric and allopatric populations of diploid and tetraploid Arrhenatherum elatius

\begin{tabular}{|c|c|c|c|c|c|}
\hline \multirow[b]{2}{*}{ Locus } & \multirow[b]{2}{*}{ Alleles } & \multicolumn{2}{|c|}{ Tetraploids } & \multicolumn{2}{|c|}{ Diploids } \\
\hline & & Parapatric (RS4) & Allopatric (SCS) & Parapatric (SZ2) & Allopatric (SGA) \\
\hline \multirow[t]{4}{*}{ GOT } & $A$ & 0.6000 & 0.6429 & 0.8611 & 0.8333 \\
\hline & $B$ & 0.4000 & 0.3095 & 0.0833 & 0.0167 \\
\hline & $C$ & 0.0 & 0.0119 & 0.0 & 0.0 \\
\hline & $D$ & 0.0 & 0.0357 & 0.0556 & 0.0 \\
\hline \multirow[t]{3}{*}{$P G I$} & $A$ & 0.8625 & 0.8333 & 0.9444 & 0.6875 \\
\hline & $B$ & 0.1125 & 0.1667 & 0.0556 & 0.3125 \\
\hline & $C$ & 0.0250 & 0.0 & 0.0 & 0.0 \\
\hline \multirow[t]{5}{*}{$L A P$} & $A$ & 0.6500 & 0.4750 & 0.7647 & 0.6080 \\
\hline & $B$ & 0.2750 & 0.4000 & 0.0588 & 0.2390 \\
\hline & $C$ & 0.0750 & 0.0875 & 0.1765 & 0.1530 \\
\hline & $D$ & 0.0 & 0.0250 & 0.0 & 0.0 \\
\hline & $E$ & 0.0 & 0.0125 & 0.0 & 0.0 \\
\hline
\end{tabular}

Table 3 Multilocus $\left(t_{\mathrm{m}}\right)$ and single-locus $\left(t_{\mathrm{s}}\right)$ outcrossing rates and rates of biparental inbreeding ( \pm standard errors) for parapatric and allopatric diploid and tetraploid populations of Arrhenatherum elatius

\begin{tabular}{lccc}
\hline & Multilocus outcrossing rate $\left(t_{\mathrm{m}}\right)$ & Single-locus outcrossing rate $\left(t_{\mathrm{s}}\right)$ & Biparental inbreeding $\left(t_{\mathrm{m}}-t_{\mathrm{s}}\right)$ \\
\hline $\begin{array}{l}\text { Diploids } \\
\text { Parapatric }\end{array}$ & $0.642(0.108)$ & $0.614(0.111)$ & \\
Allopatric & $0.930(0.087)$ & $0.903(0.075)$ & $0.027(0.020)$ \\
Tetraploids & & & $0.027(0.039)$ \\
Parapatric & $0.541(0.079)$ & $0.485(0.074)$ & $0.056(0.029)$ \\
Allopatric & $0.701(0.103)$ & $0.655(0.095)$ & $0.046(0.035)$ \\
\hline
\end{tabular}

Outcrossing rates were calculated with the MLT (diploids) and MLTET (tetraploids) computer software (Ritland, 1991) on three electrophoretic isozyme loci. Standard errors were estimated with 500 boostraps.

and when grown in a common garden, suggesting an important genetic component of flowering phenology. Tetraploids flowered earlier than diploids. In the roadside zone tetraploid plants are markedly more abundant than diploids, hence the lower seed set of the latter (compared to scree slope diploids) may be caused by pollen limitation. However, this roadside zone may not be a natural habitat for diploids hence environmental stress may act to reduce their reproductive success. Diploids from the roadside zone also flowered over a longer period than those from the scree slope. As there were no significant differences in flowering duration in controlled conditions, differences in flowering duration observed in the field may be caused by habitat stress. Scree slopes are very disturbed and dry habitats compared to the roadside zone, hence it may be unfavourable to flower over longer periods in the scree slope zone.

Several other studies have shown flowering time variation between adjacent diploid and tetraploid populations in anemophilous taxa such as Dactylis glomerata (Borrill \& Linder, 1971; Lumaret \& Barrientos, 1990; Bretagnolle \& Thompson, 1996), Plantago media (Van Dijk, 1991) and Anthoxanthum odoratum-A. alpinum (Felber, 1988). Several models can be proposed to explain such divergence. Stam (1983) showed that reproductive isolation between two populations by divergence of flowering time may be caused by environmental disturbance resulting in a nonrandom migration of genes that determine flowering time, this in turn leading to a divergence of flowering time. This model may, however, better explain ecotypic differentiation than diploid-tetra- 
ploid divergence, because the latter involves low fitness or viability of hybrids, and in consequence, weak gene exchange between cytotypes. More recently, Van Dijk \& Biljsma (1994) have shown that flowering time divergence may be a by-product of selection if the two taxa occur in patchy habitats or in a parapatric zone. In their model natural selection may shape flowering time patterns in a diploidtetraploid contact zone so that diploids and tetraploids with nonoverlapping flowering period may have a higher fitness than other plants. This model may better explain our results and those from other diploid-polyploid contact zones.

Although we observed significant flowering time divergence between diploids and tetraploids, our data provide no evidence of selection on flowering time within ploidy levels in 1994 at the El Turbon site. Although this may be caused by particular environmental pressures in the year of the experiment or by the fact that our sample sizes were too low to detect any selection (Endler, 1986), it is also likely that the divergence between the cytotypes makes any variation within cytotypes insignificant in terms of reduced hybridization with the other cytotype. Both allopatric and parapatric populations of the two cytotypes show clear divergence in flowering time in controlled conditions. Hence early flowering in tetraploids may have been established before the contact between the two cytotypes, and may actually have permitted the establishment of the zone, rather than being a consequence of selection following initial reproductive contact. Further data on a large number of parapatric and allopatric populations are necessary to distinguish between these two possibilities.

\section{Selfing in parapatric and allopatric populations}

For both ploidy levels, the two parapatric populations showed higher selfing rates than their respective allopatric populations. Results of previous studies have suggested that increased selfing may evolve to limit hybridization between closely related species (Hinton, 1976; Levin, 1985) or to reduce gene flow from nonadapted into locally adapted populations (Antonovics, 1968; Lefèbvre, 1970). In Arrhenatherum elatius, Cuguen et al. (1989) showed that lower levels of inbreeding depression can occur in metal-tolerant plants and thus facilitate increased selfing in such situations.

To our knowledge, our study is the first to show increased selfing rates in parapatric diploid and tetraploid populations compared to allopatric populations of the two ploidy levels. Several explanations can be proposed to explain the difference in mating system between allopatric and parapatric populations of the two ploidy levels.

First, increased selfing in anemophilous species may be related to abiotic or biotic factors, such as variation in density (Vaquero et al., 1989). In our study, the allopatric and parapatric populations of each cytotype occur in similar habitats (open scree slopes for the diploid populations and roadsidewoodland edge for the tetraploid populations) suggesting that environmental variation is not likely to be the cause of the selfing rate variation.

Secondly, tetraploids of Arrhenatherum elatius are known to be able to self-pollinate (Sulinowski, 1965; Cuguen et al., 1989; Ge \& Thompson, unpublished data), and in our study the difference in the outcrossing rates of allopatric and parapatric tetraploids is lower and nonsignificant compared to that between allopatric and parapatric diploids. The selfing rate of tetraploids in the parapatric zone may thus simply reffect random variation among populations in the outcrossing rate of this cytotype. In contrast, the selfing rate of the allopatric diploid population was extremely close to zero and previous studies of other allopatric diploid populations have shown no evidence of self-compatibility (Thompson \& Ge, unpublished data). Gene flow, transfer of genes increasing selfing from tetraploid to diploid plants, by back-crosses with triploids or with diploid plants formed by possible gynogenesis in tetraploids, could thus explain the higher diploid selfing rate in the contact zone. The higher selfing rate may have been subsequently maintained because of its potential adaptive significance in the contact zone. Nevertheless, the genetic data presented here, and hybrid indices calculated on the basis of variation in morphometric traits across the contact zone (Petit, Lebros \& Thompson, unpublished data) provide no evidence of introgression.

Thirdly, if gene flow between ploidy levels occurs and has a detrimental effect on seed-set, then increased selfing may occur because of a reduced contribution of outcrossed seed to the seed-set of a self-compatible plant. Hence our decreased outcrossing rates may result from a reduced (outcrossed) seed-set in the open-pollinated families we collected. However, the almost complete separation of diploid and tetraploid flowering times in the contact zone and the near lack of any selfing in the allopatric diploid population makes this an unlikely alternative.

Finally, it is possible that the higher rates of selfing have evolved in both diploids and tetraploids in situ in response to selection to limit hybridization 
with the other ploidy level. This diploid-tetraploid situation is thus analogous to metal tolerant-nontolerant populations where higher self-compatibility has been observed in the adapted populations (Antonovics, 1968; Lefèbvre, 1970). If hybridization does indeed produce inviable triploids this basis for the higher selfing rates becomes on attractive possibility and a novel occurrence of reproductive character displacement. In such cases, inbreeding depression must be low compared to selection against hybrids.

Given that almost complete flowering time divergence between diploids and tetraploids was observed, one could ask why should increased selfing be maintained? The answer to this may lie in any among-year variation in flowering time which may cause the two ploidy levels occasionally to flower at the same time. Such annual variation is a feature of other species (Rathcke \& Lacey, 1985; Dominguez \& Dirzo, 1995).

\section{Acknowledgements}

We thank C. Romero-Zarco for population location maps, J. David and J. Lepart for their helpful discussion of the work, J. Ronfort, S. Mazer, R. Lumaret, D. Schoen and F. Felber for comments on a preliminary version of this manuscript, F. Di Giusto for electrophoretic technical aid, C. Duperray at the Unity 291 of INSERM at Montpellier for help with flow cytometry, and C. Collin for assistance with plant cultivation.

\section{References}

ANTONOvics, J. 1968. Evolution in closely adjacent populations. V. Evolution of self-fertility. Heredity, 23, 219-238.

BORRILL, M. AND LINDNER, R. 1971. Diploid-tetraploid sympatry in Dactylis (Gramineae). New Phytol., 70, $1111-1124$.

BRETAGNOLLE, F. AND THOMPSON, J. D. 1996. An experimental study of ecological differences in winter growth in sympatric diploid and autotetraploid Dactylis glomerata.J. Ecol., 84, 343-351.

CUGUen, J., ACHEROY, M., LOUTFl, A. L., PETIT, D. AND VERNET, P. 1989. Breeding system differentiation in Arrhenatherum elatius populations: evolution toward selfing? Evol. Trends Plants, 3, 17-24.

DOBZHANSKY, т. 1940. Speciation as a stage in evolutionary divergence. Am. Nat., 74, 312-321.

DOMINGUEZ, C. A. AND DIRZO, R. 1995. Rainfall and flowering synchrony in a tropical shrub: variable selection on the flowering time of Erythroxylum havanense. Evol. Ecol., 9, 204-216.
ENDler, J. A. 1986. Natural Selection in the Wild. Princeton University Press, Princeton, NJ.

FELBER, F. 1988. Phénologie de la floraison de populations diploïdes et tétraploïdes d'Anthoxanthum alpinum et d'Anthoxanthum odoratum. Can. J. Bot., 66, 2258-2264.

FELBER, F. 1991. Establishment of a tetraploid cytotype in a diploid population: effect of relative fitness of the cytotypes. J. Evol. Biol., 4, 195-207.

HINTON, W. F. 1976. Introgression and the evolution of selfing in Calyptridium monospermum (Portulacaceae). Syst. Bot., 1, 85-90.

HOWARD, D. J. 1993. Reinforcement: origin, dynamics, and fate of an evolutionary hypothesis. In: Harrison, R. G. (ed.) Hybrid Zones and the Evolutionary Process, pp. 46-69. Oxford University Press, New York.

LANDE, R. AND ARNOLD, s. J. 1983. The measurement of selection on correlated characters. Evolution, 37, 1210-1226.

LEFĖBVRE, C. 1970. Self-fertility in maritime and zinc mine populations of Armeria maritima (Mill.) Willd. Evolution, 24, 571-577.

LEVIN, D. A. 1975. Minority cytotype exclusion in local plant populations. Taxon, 24, 35-43.

LEVIN, D. A. 1985. Reproductive character displacement in Phlox. Evolution, 39, 1275-1281.

LUMARET, R. AND BARRIENTOS, E. 1990. Phylogenetic relationships and gene flow between sympatric diploid and tetraploid plants of Dactylis glomerata (Gramineae). Pl. Syst. Evol., 169, 81-96.

MITCHELL-OLDS, T. AND SHAW, R. G. 1987. Regression analysis of natural selection: statistical inference and biological interpretation. Evolution, 41, 1149-1161.

$\mathrm{NE1}$, M. 1978. Estimation of average heterozygosity and genetic distance from a small number of individuals. Genetics, 89, 583-590.

PFITZENMEYER, C. D. C. 1962. Biological Flora of the British Isles. Arrhenatherum elatius (L.) J. and C. Presl. J. Ecol., 50, 235-245.

RATHCKE, B. AND LACEY, E. P. 1985. Phenological patterns of terrestrial plants. Ann. Rev. Ecol. Syst., 16, 179-214.

RITLAND, K. 1990. A series of FORTRAN computer programs for estimating plant mating systems. J. Hered., 81, 235-237.

RITLAND, K. 1991. MLTET. Generalized program for estimating inbreeding parameters in tetraploids. University of Toronto, Toronto, Canada.

SAS, 1990. SAS/sTat Users guide. Cary, N.C.

SCHLUTER, D. 1990. GLMS, version 3. University of British Columbia, Vancouver, Canada.

STAM, P. 1983. The evolution of reproductive isolation in closely adjacent plant populations through differential flowering time. Heredity, 50, 105-118.

SULINOWSK1, s. 1965. Variation in forms and biology of flowering in Arrhenatherum elatius (L.) P.B. Part II. Effects of self- and cross-pollination on seed setting in Arrhenatherum elatius. Genetica Polonica, 6, 292-312.

SWOFFORD, D. L. AND SELANDER, R. B. 1981. BIOSYS-1. $A$ computer program for the analysis of allelic variation in genetics. Release 1. University of Illinois, Urbana, IL. 
THOMPSON, J. D. AND LUMARET, R. 1992. The evolutionary dynamics of polyploid plants: origins, establishment and persistence. Trends Ecol. Evol., 7, 302-307.

VAN DIJK, P. 1991. Evolutionary Aspects of Polyploidy in Plantago media L. Ph.D. Thesis, University of Groningen.

VAN DIJK, P. AND BIJLSMA, R. 1994. Simulations of flowering time displacement between two cytotypes that form inviable hybrids. Heredity, 72, 522-535.
VAQUERO, F., VENCES, F.J., GARCIA, P., RAMIREZ, L. AND PEREZ DE LA VEGA, M. 1989. Mating system in rye: variability in relation to the population and plant density. Heredity, 62, 17-26.

ZIMMERMAN, M. AND GROSS, R. s. 1984. The relationship between flowering phenology and seed set in an herbaceous perennial plant, Polemonium foliosissimum Gray. Am. Midl. Nat., 111, 185-191. 